Acta Crystallographica Section D

Biological Crystallography

ISSN 0907-4449

\section{The care and nurture of undulator data sets}

\author{
D. Flot, ${ }^{\text {b }}$ E. J. Gordon, ${ }^{a}$ \\ D. R. Hall, ${ }^{a}$ G. A. Leonard, ${ }^{a}$ \\ A. McCarthy, ${ }^{b}$ J. McCarthy, ${ }^{a}$ \\ S. McSweeney, ${ }^{\text {a* E. Mitchell, }}{ }^{\mathrm{a}}$ \\ D. Nurizzo, ${ }^{a}$ R. G. B. Ravelli ${ }^{b}$ \\ and W. Shepard ${ }^{\mathrm{a}}$
}

aESRF, 6 Rue Jules Horowitz, 38043 Grenoble, France, and ${ }^{\mathbf{b}} \mathrm{EMBL}, 6$ Rue Jules Horowitz,

38042 Grenoble, France

Correspondence e-mail: seanmcs@esrf.fr
Undulator radiation is the X-ray source of choice for modern macromolecular crystallography beamlines. Here, the basic properties of undulator sources are described and it is indicated why they make such good X-ray sources for macromolecular crystallography. Collection of excellent data from these beamlines is not always straightforward; therefore, a number of rules are postulated for undulator data collection and guidelines are offered which will help to ensure a satisfactory experiment.

\section{Introduction}

Macromolecular structure determination is increasingly carried out at synchrotron-radiation (SR) sources. The last decade has seen the rate of deposition of macromolecular structures in the PDB grow exponentially. Linked to this trend is the development of undulator radiation as the $\mathrm{X}$-ray source that is best adapted to the needs and requirements of the macromolecular crystallography (MX) experiment.

Originally, synchrotron-based MX was undertaken on beamlines fed by X-ray sources that exploited the radiation emitted from bending magnets or wigglers (wavelength shifters). These sources produce broad fans of radiation (typically many millimetres or milliradians in size) and are well adapted to the installation of many experimental facilities on the same source. However, the relatively high X-ray beam divergence and the large X-ray beam (or electron-source) size present a number of technical difficulties which make the provision of tightly focused X-ray beams problematic.

With the construction of third-generation high-energy sources, it became practical to construct undulator sources producing photons in the energy range appropriate for MX diffraction experiments. With experience in the taming of these sources, the reliability and optimization of beamlines for MX has resulted in the undulator becoming the X-ray source of choice for modern MX beamlines.

Undulator sources can produce extremely high-intensity and well collimated X-ray beams that deliver many photons through a sample and thereby greatly enhance the possibility of achieving higher resolution diffraction data. Indeed, access to these facilities has breathed new life into many projects which were otherwise moribund or considered impossible. However, such a high dose of X-rays brings with it additional complications that can render measurements problematical. It is thus important that the experimenter displays appropriate care and foresight when planning and undertaking the diffraction experiment.

In this paper, we discuss the creation and properties of undulator radiation and consider the characteristics of the $\mathrm{X}$-rays in relation to the experiment to be performed. The
Received 2 March 2005 Accepted 11 October 2005
(C) 2006 International Union of Crystallography Printed in Denmark - all rights reserved 
Table 1

Electron-beam and X-ray beam properties for the insertion devices available at the ESRF.

\begin{tabular}{lll}
\hline & \multicolumn{2}{l}{ ID section } \\
\cline { 2 - 3 } & $\begin{array}{l}\text { Even } \\
(\text { ID2, ID6, } \ldots)\end{array}$ & $\begin{array}{c}\text { Odd } \\
(\text { ID1, ID3, } \ldots)\end{array}$ \\
\hline Horizontal r.m.s. electron-beam size $(\mu \mathrm{m})$ & 402 & 59 \\
Horizontal r.m.s. electron divergence $(\mu \mathrm{rad})$ & 10.7 & 90 \\
Horizontal beam size at $25 \mathrm{~m}(\mu \mathrm{m})$ & 670 & 2310 \\
Vertical r.m.s. electron-beam size $(\mu \mathrm{m})$ & 7.9 & 8.3 \\
Vertical r.m.s. electron divergence $(\mu \mathrm{rad})$ & 3.2 & 3 \\
Vertical beam size at $25 \mathrm{~m}(\mu \mathrm{m})$ & 88 & 83 \\
\hline
\end{tabular}

experience of observing many thousands of undulator experiments leads us to propose some 'rules' to be followed when undertaking a MX experiment. These rules are amplified with examples taken from the use of ESRF beamlines. We conclude with a discussion of the perspectives for the development and use of undulator beamlines in the future.

\section{Undulator radiation sources and experimental considerations}

A detailed description of the production of synchrotron light from undulator sources is beyond the remit of this paper. The

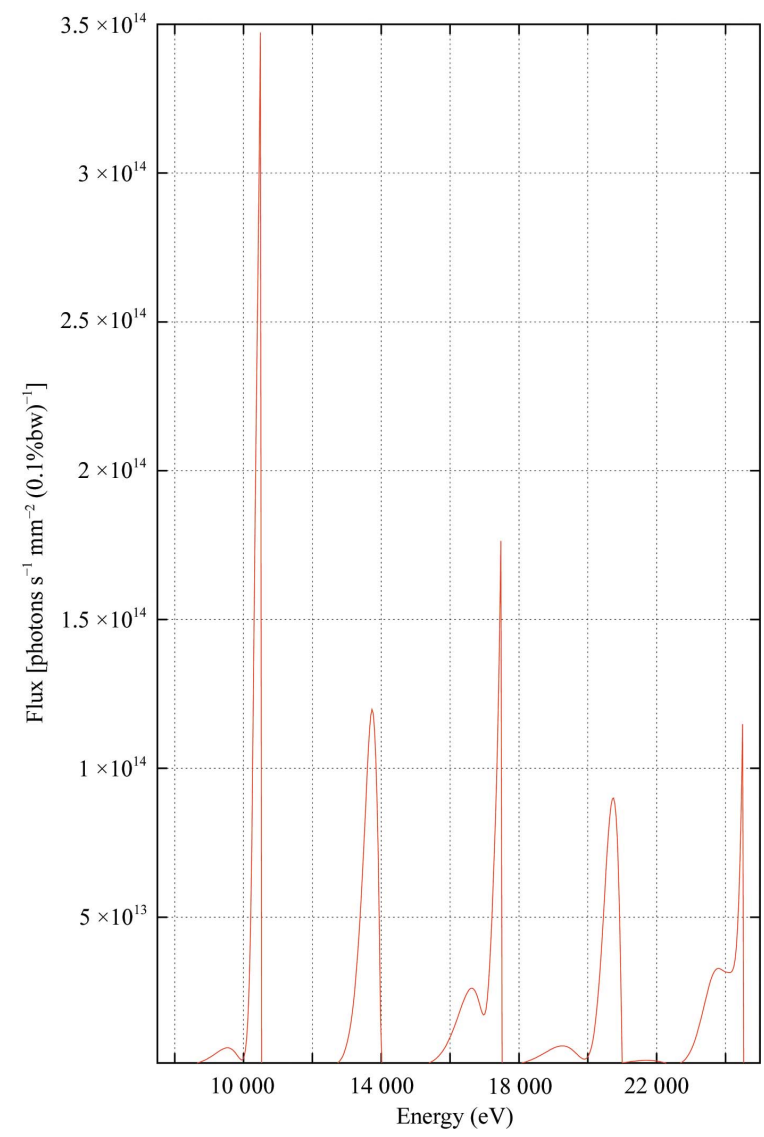

Figure 1

Typical undulator spectrum from an ESRF U35 undulator. Flux through a $1 \mathrm{~mm}^{2}$ aperture at $25 \mathrm{~m}$ from the source. Source properties as indicated in Table 1 (even ID). Calculated using the URGENT code (Walker \& Diaviacco, 1992) as implemented in XOP (Sanchez del Rio \& Dejus, 1997). interested reader is referred to Winick (1995) for a full account. Our intention here is only to highlight those properties of the source that are important to the MX experiment.

Undulator radiation is emitted when relativistic electrons traverse a periodic magnetic lattice. The magnetic field causes small oscillations in the electron trajectory and at the peak of each oscillation light is emitted. Providing that the extent of these oscillations is small, the light will constructively interfere and the number of photons emitted within a small solid angle is increased dramatically.

For the experimenter, the light emitted has several important characteristics: the light is constrained into a small solid angle and has very low divergence (see Table 1 for source and $\mathrm{X}$-ray beam properties of ESRF undulators). These properties mean that the X-ray beam can be delivered to a small area, enabling extremely small samples to be examined. The low divergence also makes practical the investigation of samples with large unit cells. In addition, the interference between successive undulations results in a spectrum containing peaks at certain energies (Fig. 1) and the position of these peaks may be altered by varying the gap between the elements of the magnetic structure, thereby enabling many anomalous scattering experiments to be undertaken using undulator radiation.

The same properties that make undulator sources so attractive for MX also make them very unforgiving to experimental mistakes. It is the technique of utilizing these sources to maximum advantage that is the subject of the rest of this paper.

\section{Rules for data collection at undulator beamlines}

The rules described below arise from our experience of data collection on undulator beamlines. However, many if not all of the rules described will apply to any MX data-collection experiment.

\subsection{Rule 1: Do the simple things properly}

A good experiment at a SR source starts before one leaves the home laboratory. The equipment brought can have an enormous effect on the quality of the data collected: ensure that all equipment is of high quality and correctly maintained. Goniometer heads should be in good working condition. Importantly, before cryocooling crystals one should verify that the cryoloops in which the crystals are to be cryocooled are in good condition, are of an appropriate size for the sample and do not have an excessively long stem between the pin and the loop. Verify that the loop has been properly attached to the pin onto which it is mounted. The difference in data quality when using 'good' or 'bad' loops in which to cryocool crystals is shown in Fig. 2.

It can be seen that using a 'poor' loop results in data with an $R_{\text {sym }}$ that is $2 \%$ worse in all resolution bins than it is for data collected using a crystal in a 'good' loop. This deterioration in data quality is almost certainly a result of increased crystal vibration in the loop with a longer stem. During image 
processing with MOSFLM, the program reported slippage of the crystal and the orientation matrix was refitted several times during the processing of 100 images.

Probably the most common cause of crystal vibration during data collection is the misalignment of the cryostream used to maintain the crystal at a constant temperature (usually $\sim 100 \mathrm{~K}$ ) during data collection. Here, the term 'misalignment' is used to refer to the fact that the crystal is not at the centre of the steam of gaseous nitrogen or to the fact that the nozzle of the cryostream is too far from the crystal itself. Both result in the flow of nitrogen over the crystal becoming turbulent. Tests of the effects of such misalignments have been carried out at many MX beamlines at the ESRF.

To demonstrate the systematic effect of cryostream-crystal distance on data quality, several analogous complete data sets were collected from the same single crystal of bovine trypsin with the end of the nozzle of the cryostream placed consecutively $5,10,15$ and $5 \mathrm{~mm}$ from the sample.

As can be seen from Fig. 3, when the cryostream was $5 \mathrm{~mm}$ from the crystal data quality was very good. Increasing the cryostream-crystal distance to $10 \mathrm{~mm}$ increases $R_{\text {sym }}$ by around $2 \%$ in all resolution bins and when the cryostream was placed at $15 \mathrm{~mm}$ from the sample $R_{\text {sym }}$ increases (relative to a distance of $5 \mathrm{~mm}$ ) by around $7-8 \%$ in all resolution bins. Clearly, this latter data set is of very poor quality and, particularly for a MAD/SAD data collection, would probably prove fatal to the aims of the experiment. A similar deterioration in data quality is seen when the flow of nitrogen over the crystal is turbulent. However, we have not observed such marked effects when using lithographic loops or where the stalk of the loop is short or stiffened with glue (unpublished results).

Properly centring the crystal to the X-ray beam can have dramatic effects on the quality of the data observed; this error

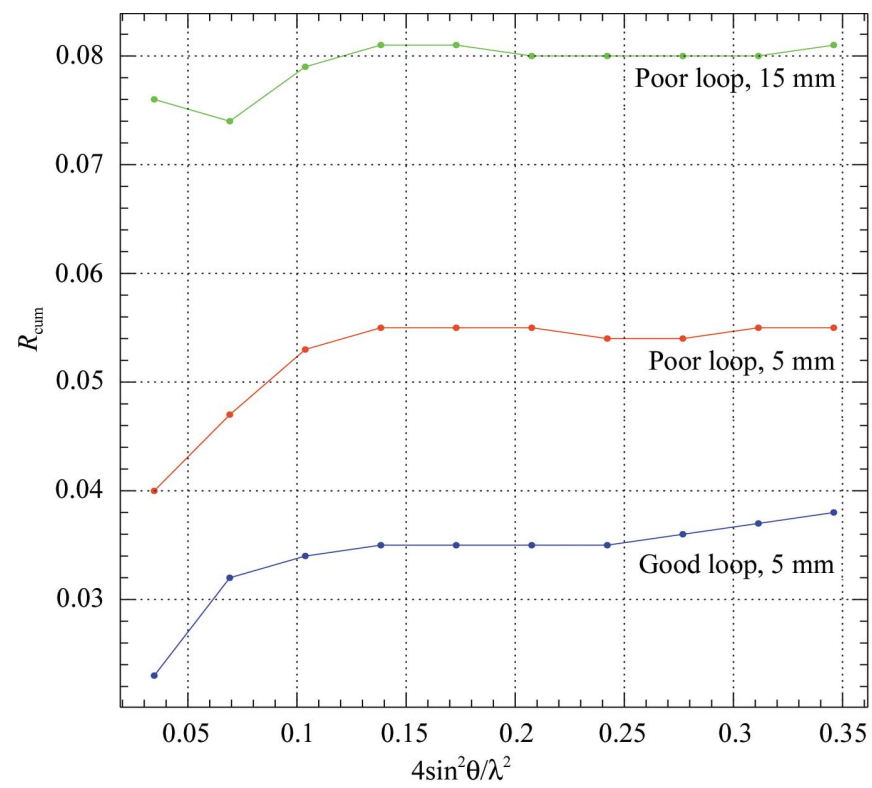

Figure 2

Effect of both cryostream alignment and cryopin quality on the data observed. seems to cause more problems at lowest resolutions. If a sample is small and not well centred, the scattering volume of the crystal illuminated by the small beams of undulator sources can vary and give rise to deleterious effects on the data. Note that poor centring of crystals can lead to the appearance that a crystal is suffering from radiation damage and lead to the early termination of data collection from a well diffracting sample!

Finally, it is often the case that in one orientation the crystal appears to diffract strongly whilst in the another it diffracts weakly. It is therefore wise to test diffraction in orthogonal orientations.

\subsection{Rule 2: Have a plan, but don't stick to it slavishly!}

Somewhat paradoxically, the fact that modern undulatorbased MX beamlines allow the collection of many diffraction data sets in a single day often leads to their inefficient use. The speed at which data are collected on such beamlines means that experimenters often do not have sufficient time to think about what to do next, leading to mistakes being made and a non-optimal use of beamtime. It is thus important to have made a plan of how beamtime will be used before arriving on the beamline.

As a minimum, a plan should define the overall scientific priorities as well as a collection of minimum requirements (a 'diffraction plan') for each sample to be studied. Make sure that these priorities are agreed beforehand, especially if the crystals belong to different team members. If there are crystals to test, decide beforehand how many to test before moving on to the next project. Ensure that you have contact details for team members who are not at the synchrotron in case of unexpected results.

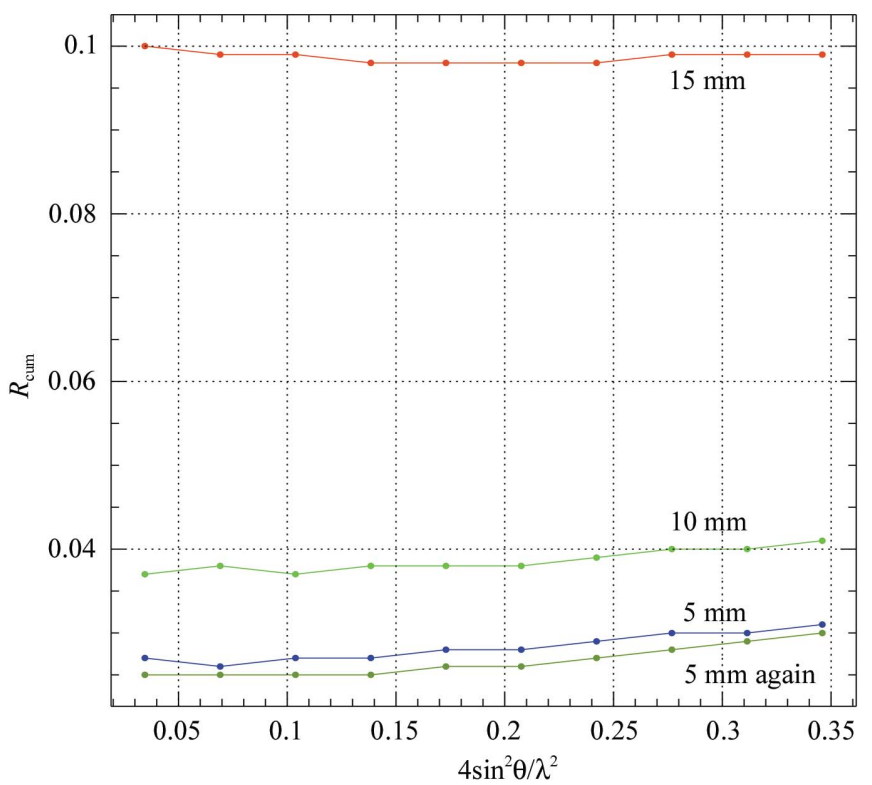

Figure 3

The effect of cryostream position on data quality. Each data point represents a complete orthorhombic trypsin data set. 
Information that the plan should include is as follows.

(i) The type of the data collection to be performed and its aims [single wavelength for molecular replacement (MR); MAD/SAD data collection, screening for heavy atoms etc.]. This information helps to determine the quality of data that one needs to collect. For example, a much poorer crystal and diffraction quality (as measured in terms of $R_{\text {sym }}$ ) can be tolerated when a data set is collected for use in MR than for use with MAD/SAD. For a MAD/SAD data collection the substituted heavy atom needs to be known.

(ii) Basic crystallographic information (unit-cell parameters and space group, previously observed and desired diffraction limits).

How data collections should then proceed depends upon a proper characterization of each sample at the beamline coupled with a knowledge of the characteristics of the beamline being used. The former can often be carried out automatically (c.f. the DNA collaboration; Leslie et al., 2002) and the predicted data-collection strategies (including exposure time) and diffraction limits obtained are a reliable guide. However, such protocols do not yet include the inevitability of radiation damage to crystals and exposure times in particular may have to be modified to take this into account. A degree of flexibility on the part of the experimenter is thus required. Process data as you collect it; if you see data quality deteriorate, do not be afraid to stop data collection and start again with a new crystal, this time with attenuation. If you have doubts about crystal characterization, err on the side of low symmetry.

Low-resolution reflections are particularly susceptible to overloading (or saturating) the detector. These reflections can play a critical role in the success of any experiment and reasonable estimates of the intensity must be obtained. If the exposure time leads to a large number of overloaded reflections, it is advisable to collect a complete low-resolution data set (with a heavily attenuated X-ray beam) before proceeding with other data collection.

MAD/SAD experiments on tuneable beamlines may require even more flexibility and a number of questions need to be asked both before and during such data collections. Those to be asked before data collection begins include the following: should one collect to the diffraction limit of the crystals? How many wavelengths should be collected? Which wavelength should be collected first? Is it necessary to scan the absorption edge before collecting data? Those questions to be asked after data collection starts include: when should data collection be stopped?

None of these questions are straightforward to answer. However, our experience suggests in the first instance that the following general approach is worthwhile.

(i) Be conservative! Collect data to a resolution that is lower than the diffraction limit of the crystal. A reasonable cutoff point would be where the $R_{\text {sym }}$ for a resolution shell is more than $10 \%$ [use BEST (Popov \& Bourenkov, 2003) to predict this]. Do not be afraid to add attenuation, especially if this action also avoids saturated low-resolution reflections. (ii) Firstly, collect a complete SAD data set on the highenergy side of the absorption edge (high-energy remote) in order to determine whether a heavy atom is bound to the protein or not. Often for SeMet-substituted crystals this data set will be sufficient to allow structure solution without resorting to a multiwavelength approach (Dauter, 2002).

(iii) Integrate, reduce and analyse data as it is being collected. Collect the first wavelength until you have at least a multiplicity of six (Leonard et al., 2005).

Programs such as XPREP, SHELXD and SHELXE (Schneider \& Sheldrick, 2002; Sheldrick, 2002) can very quickly give indications as to whether there is anomalous signal in the data, whether the heavy-atom substructure can be determined and whether the data already collected will result in structure solution. In cases where it is clear that a structure can be solved with single-wavelength data, it is probably best to continue collecting at this wavelength (see Dauter \& Adamiak, 2001; Ramagopal et al., 2003; Usón et al., 2003 for the effects of multiplicity on quality of phases) rather than to carry out full a MAD experiment. Additionally, failure to determine the heavy-atom substructure at this stage probably means that either the data quality is not good enough or the heavy atom is not bound. In these cases it is often better to abandon further data collection and to search for a crystal giving better quality diffraction.

\subsection{Rule 3: Speed kills}

A strong temptation at an undulator beamline is to use the high-intensity beam to collect data rapidly by using very short exposure times. The combination of extremely high intensity with air-bearing based spindles allows exposure times of tens of milliseconds. This temptation should not be given in to since speed can damage the data in a number of ways. In particular, the mechanics of the current shutters used for data collection are neither fast nor accurate enough for sub-100 ms (or sometimes even sub-second) exposure times. Accurate spindle-shutter synchronization is absolutely essential for good data quality since the crystal should only be exposed and data collected once the spindle is moving at constant speed and the opening and closing times must correspond to the expected oscillation range. The shutter also needs to have reproducible opening and closing times so that the exposure time for each oscillation is constant. One important issue therefore in defining a minimum exposure time for data collection is the type of shutter used and its mechanical characteristics. There is a (significant) delay between the shutter receiving the signal to open or close and the moment where it is actually fully open or closed. For currently available fast shutters these delays vary between $\sim 3 \mathrm{~ms}$ (piezo shutters) and more than $30 \mathrm{~ms}$ (mechanical shutters) and, depending on the shutter design, the reproducibility of this lag may also vary. Even for the fastest shutters, a $3 \mathrm{~ms}$ error in a $100 \mathrm{~ms}$ exposure can lead to a $6 \%$ error and will show up particularly in the measurement of the partial reflections. Low-resolution data is often the worst affected since these reflections remain in the 
Bragg condition for longer and are therefore mostly collected as partials.

Since data-collection rates of 20-30 images per minute are possible with standard $1 \mathrm{~s}$ exposure times, another temptation is to 'just collect'. Once again this must be avoided if good data quality is to be expected. Rapid data collection should not be confused with rabid data collection: collecting poor data quickly is not desirable! On an undulator beamline it is very important to take a few minutes to develop a plan to collect the data and to ensure that the experiment is correctly set up. The plan to collect the data should take into account the type of experiment to be carried out: native, high resolution, MAD, SAD. For example, for a MAD data collection the experiment is more likely to be successful if the data are complete and redundant at all wavelengths measured, so the crystal will need to last in the beam. Since radiation damage is an important factor on high-intensity beamlines, such an experiment will only be successful if the beam is attenuated accordingly. The time spent doing this will be saved later since you are more likely to collect good data the first time round.

\subsection{Rule 4: All crystals die}

Use of the brightest undulator sources has reignited interest in the prospect and effects of damage induced by irradiation with X-rays. Numerous studies (Burmeister, 2000; Ravelli \& McSweeney, 2000; Weik et al., 2000) have shown that specific structural changes are induced in the sample under study in addition to other non-specific damage. When planning an experiment, the experimenter should keep in mind the likely consequences of prolonged exposure to X-rays. The direct consequences and their interpretation will also depend upon the type of experiment being performed.

A number of recent studies have shown that specific changes are induced in the anomalous scattering substructure during MAD and SAD experiments (Rice et al., 2000; Zwart et al., 2004; Weiss et al., 2004; Ravelli et al., 2005). In an experiment where the goal is to amass phasing information, the primary aim is to keep the anomalous signal in place during the experiment. Practically, this means that the minimum dose required to achieve the experimental aims should be used. From Ravelli et al. (2005), we include Fig. 4 to demonstrate the effect of radiation damage on anomalous difference Pattersons. In order to assess the onset of deleterious (to the anomalous signal) damage, it is prudent to start data processing as soon as possible in order to track merging statistics and any anomalous signal. Powerful packages are available nowadays that can determine the substructure within minutes (Schneider \& Sheldrick, 2002). The behaviour of these programs as a function of redundancy is a very good marker of the amount of specific damage to the anomalous scatterers (Ravelli et al., 2005; Leonard et al., 2005). In the absence of radiation damage, the success rate and the quality of the substructure should improve with redundancy. In the presence of radiation damage, the useful anomalous signal will be gradually worn away and redundancy will reduce the success of the substructure determination.

It is likely that for MR and substrate-binding studies radiation damage is less of an issue. MR requires complete data, especially at low resolution, and special care has to be taken to avoid saturated pixels on the detector in this region. Thus MR, like anomalous dispersion experiments, will benefit from a careful reduction in the dose used to collect a complete data set. It has been noted (Ravelli \& McSweeney, 2000) that small but signification molecular rotations and translations may occur as a result of irradiation; the details of biases induced by this process require further analysis.

A number of programs are available to allow a realistic estimate of the total exposure time required for a data collection (Murray et al., 2004). The radiation-lifetime estimate made by these programs does not take specific damage into account and it might be that the susceptibility of some crucial crystal-contact residues could result in an enhanced sensitivity of the crystal (Murray et al., 2005). However, in general all crystals seem to have, at least within an order of magnitude, a similar radiation-dose lifetime and it is wise to

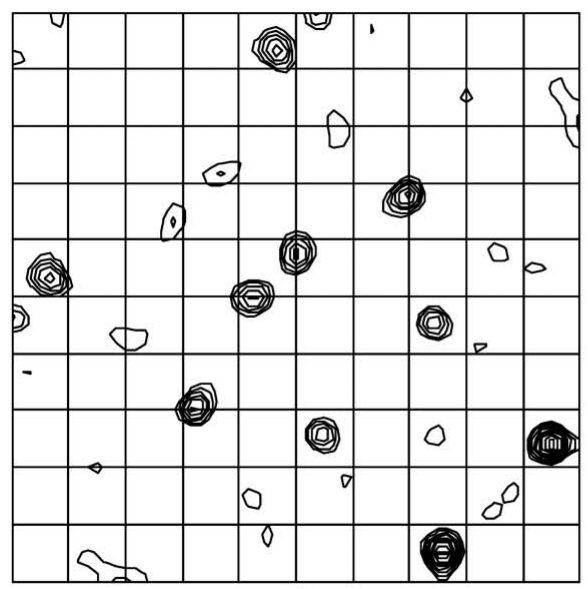

(a)

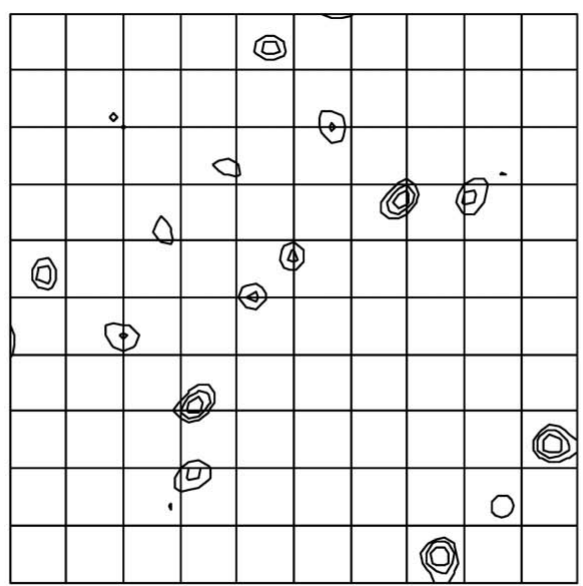

(b)

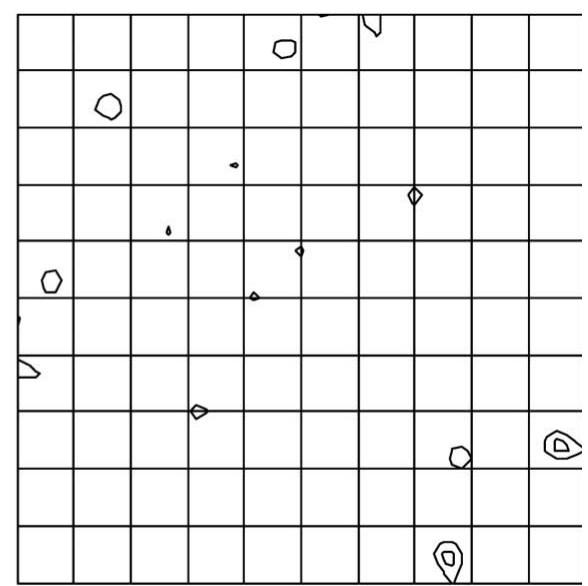

(c)

Figure 4

The change in anomalous signal as a function of X-ray dose. The dose received in $(b)$ is the equivalent of three times the dose in $(a)$ and for $(c)$ the dose is four times that in (a). Full details are available in Ravelli et al. (2005). 
translate this into a time for the total exposure that can be used for a given crystal on a given beamline. For the ESRF undulator MAD beamlines, a rule-of-thumb number would be 100-1000 s of unattenuated X-ray exposure.

Recent advances in the data-collection strategy program BEST (Popov \& Bourenkov, 2003) make it possible to train strategy software with the decay behaviour of a specific crystal at a certain beamline. Thus, for the moment, it is advisable that the experimenter uses the minimum possible X-ray dose consistent with the aims of the experiment. In the future it is possible that computer programs will be able to accurately assess the exposure time available to a given crystal and exploit the information content within the radiation damage as demonstrated initially by Ravelli et al. (2003) and subsequently by others (Evans et al., 2003; Schiltz et al., 2004). The future remains bright, but in the meantime it pays to be cautious.

\subsection{Rule 5: Process as you collect}

There are many problems with a data collection that can be remedied if they are identified early. With modern dataprocessing programs it is simple to process your data as you are collecting it and, as stated earlier, this should be a mandatory part of the experiment.

\subsection{Rule 6: Not all bad data are useless}

During the CCP4 meeting (Reading, January 2005) many references were made to the issue of 'bad data'. Examples included data from twinned crystals, incomplete data at high resolution, weak diffraction, data that suffered from radiation damage, Laue data and data that suffered from 'shuttersputter'.

The use of the phrase 'bad data' refers to, in our opinion, whether data are useful or not. The criterion 'useful', however, is a rather subjective one. There are numerous examples where data can be treated more or less automatically, leading to a structure with minimal intervention. Are these data referred to as 'good', in contrast to those that are resistant to automated treatment? This may well prevent structural biologists, especially those who are new to the field, spending time on data that are challenging for one reason or another but that would otherwise result in a structure determination.

Recorded intensities have to be significant, meaning that diffracted beams should give a measurable number of counts above the experimental background. As long as this criterion is fulfilled, the data can be called 'good'. Whether the data are useful depends on many things, not the least the software used. For example, some packages can handle twinned data, whereas others cannot. Radiation damage is traditionally corrected for using overall scale and $B$ factors alone, whereas only recently have more sophisticated models been investigated (Diederichs et al., 2003; Kabsch, 1988). The IUCr has evaluation criteria for defining high-quality data (values of $R_{\text {sym }}<20 \%$ ); however, some programs can make excellent use of data that do not fulfill these criteria, allowing one for example to autobuild a structure. Many synchrotrons are nowadays equipped with square CCD-mosaic detectors. Data that are collected in the corners of these detectors are useful if high resolution is required, even though the completeness and redundancy will be worse than for the data collected in the central area of the detector. Laue data are in general noisier than monochromatic data owing to the increased polychromatic background; however, such data may be useful if a subsecond time resolution is required for kinetic enzymatic studies at room temperature. Finally, shutter-sputter could be monitored in parallel to the X-ray data collection (Arzt et al., 2005 ) and in principle it is possible to correct for it during data processing. Such software will be developed for futuregeneration detectors where the detector is read semicontinuously, leaving very small time windows where the data are being read out while the crystal is still rotating and exposed to the X-ray beam.

However time-efficient it is for most structural biologists to move quickly to another crystal and data set when the original one gave some kind of problem, we hope that some of our readers will be interested in turning significant data that are not useful today into data that are useful tomorrow.

\section{Perspectives}

With the role of macromolecular crystallography expanding rapidly as a standard research tool for academia and industry alike, fewer and fewer scientists are experienced in the use of synchrotron radiation. To a great extent, implementation of the rules outlined above in an automatic 'beamline wizard' could help remove a source of uncertainty (the user) from an undulator synchrotron beamline measurement.

Many experimental issues can be managed by automated hardware (for example, sample-mounting robots) or software [for example, indexing and strategy (Leslie et al., 2002) and beamline alignment (Arzt et al., 2005)]. These are under development at many synchrotrons. All of the ESRF MX beamlines are expected to be equipped with sample changers, high-precision goniometers and an updated software-control environment by the end of 2005. Once the experimental steps are integrated, including the routing of sample information from crystallization robot or home-laboratory databases, it becomes simpler to feed back, in real time, the quality of data in terms of radiation damage and structure solution.

One of the mistakes made most often and amongst the most debilitating is overexposing a crystal to the X-ray beam (\$3.2). Radiation damage might be able to be correctd for by postprocessing (Diederichs et al., 2003) and/or used in phasing (Ravelli et al., 2003; Evans et al., 2003; Schiltz et al., 2004). Work is under way to better understand radiation damage and use it positively.

The success of macromolecular crystallography has contributed to the development of fast and accurate X-ray detectors, where X-ray detection has advanced from film to CCD-based detectors in a short time. The exposure time required on undulator beamlines can be minimal and a new generation of X-ray detectors are required and under development. Soon it may be possible to operate in continuous 
rotation mode where data images are collected with dead times sufficiently small that continuous sample rotation is feasible (Brönnimann et al., 2004). Advances in this technology would eliminate the errors introduced with shutter and rotation synchronization (\$3.3). With new detectors, the full power of the undulators could be used with no attenuation, thereby making data collections possible in seconds.

While we wait for the next generation of X-ray detectors, there are a number of recent developments in place or near completion worth mentioning. One immediate success of the new high-precision goniometers at the ESRF has been the incorporation of a high-speed inverse-beam mode for data collection, enabling the Friedel paired reflections to be collected close in time, and preliminary results have been encouraging (Ravelli \& McCarthy, personal communication). When spindles with high rotation speeds are combined with a mini- $\kappa$ system for reorienting crystals along a chosen axis, allowing the Friedel pairs to be collected on the same image, the goniometry becomes yet more useful. The mini- $\kappa$ system may also allow the anomalous phasing signal arising from anisotropic anomalous scattering to be maximized (Bricogne et al., 2005), although this will require some study.

Although undulator beams when focused are relatively small (of the order of 50-100 $\mu \mathrm{m}$ ), there is strong demand for 'microfocus' beams. The prevalence of small crystals arises partly from the trend to study increasingly complex systems, which tend to give small crystals containing few diffracting units. Suitable focusing elements to achieve a 1-20 $\mu \mathrm{m}$ X-ray beam include Kirkpatrick-Baez geometry mirrors, compound refractive or Fresnel lenses. It has been demonstrated that the combination of a micrometre-sized beam with a dedicated instrument, such as the EMBL-ESRF micro-diffractometer, can allow data collection from micrometre-sized crystals (Perrakis et al., 1999), where previously it was only possible to study such small crystals by electron diffraction. The increased flux in a micro-focus beam can compensate for the decreasing scattering volume, but at the cost of a much higher dose rate and increasing radiation damage. With source improvements, the possibility arises of generating very highly focused, very highly intense X-ray beams of nano-dimensions. This could allow the investigation of accordingly minute samples which would challenge the currently held view of protein crystallography. Issues such as beam/sample stability in time and space would need to be resolved as well as the challenge of using 'diffraction' data collected from samples with fewer than $10^{9}$ diffracting units.

Small beams are not only useful for microcrystals but also for large crystals that contain a molecule that is particularly sensitive to radiation damage or that are inhomogeneous. The small beam permits the data-collection strategy to be modified to expose only a small part of the crystal and then translate the sample to expose a fresh part of the crystal in order to collect a complete data set. With the use of such small samples comes the requirement to develop sample-handling techniques, perhaps by looking at small-molecule crystallography or novel sample supports.

\section{Conclusions}

Developments at synchrotrons are continuing apace to allow users to benefit from the intense X-ray sources available. Undulator sources remain the best source of X-rays for macromolecular crystallography. With care and the application of the rules described here, they can be tamed to allow even the most difficult problem to successfully tackled.

\section{References}

Arzt, S. et al. (2005). Prog. Biophys. Mol. Biol. 89, 124-152.

Bricogne, G., Capelli, S., Evans, G., Mitschler, A., Pattison, P., Roversi, P. \& Schiltz, M. (2005). J. Appl. Cryst. 38, 168-182.

Brönnimann, C., Bühler, C., Eikenberry, E., Horisberger, R., Hülsen, G., Schmitt, B., Schulze-Briese, C., Suzuki, M., Tomizaki, T., Toyokawa, H. \& Wagner, A. (2004). Synchrotron Radiat. News, 17, 23-30.

Burmeister, W. (2000). Acta Cryst. D56, 328-341.

Dauter, Z. (2002). Acta Cryst. D58, 1958-1967.

Dauter, Z. \& Adamiak, D. A. (2001). Acta Cryst. D57, 990-995.

Diederichs, K., McSweeney, S. \& Ravelli, R. (2003). Acta Cryst. D59, 903-909.

Evans, G., Polentarutti, M., Djinovic Carugo, K. \& Bricogne, G. (2003). Acta Cryst. D59, 1429-1434.

Kabsch, W. (1988). J. Appl. Cryst. 21, 916-924.

Leonard, G. A., Sainz, G., de Backer, M. M. E. \& McSweeney, S. (2005). Acta Cryst. D61, 388-396.

Leslie, A. G. W., Powell, H. R., Winter, G., Svensson, O., Spruce, D., McSweeney, S., Love, D., Kinder, S., Duke, E. \& Nave, C. (2002). Acta Cryst. D58, 1924-1928.

Murray, J. W., Garman, E. F. \& Ravelli, R. B. G. (2004). J. Appl. Cryst. 37, 513-522.

Murray, J. W., Rudino Pinera, E., Owen, R. L., Grininger, M., Ravelli, R. B. G. \& Garman, E. F. (2005). J. Synchrotron Rad. 12, 268-275.

Perrakis, A., Cipriani, F., Castagna, J., Claustre, L., Burghammer, M., Riekel, C. \& Cusack, S. (1999). Acta Cryst. D55, 1765-1770.

Popov, A. N. \& Bourenkov, G. P. (2003). Acta Cryst. D59, 1145-1153.

Ramagopal, U. A., Dauter, M. \& Dauter, Z. (2003). Acta Cryst. D59, 1020-1027.

Ravelli, R., Leiros, H., Pan, B., Caffrey, M. \& McSweeney, S. (2003). Structure, 11, 217-224.

Ravelli, R. \& McSweeney, S. (2000). Structure Fold. Des. 8, 315-328.

Ravelli, R. B. G., Nanao, M. H., Lovering, A., White, S. \& McSweeney, S. (2005). J. Synchrotron Rad. 12, 276-284.

Rice, L., Earnest, T. \& Brunger, A. (2000). Acta Cryst. D56, 14131420.

Sanchez del Rio, M. \& Dejus, R. J. (1997). Proc. SPIE, 3152, 148-157. Schiltz, M., Dumas, P., Ennifar, E., Flensburg, C., Paciorek, W., Vonrhein, C. \& Bricogne, G. (2004). Acta Cryst. D60, 1024-1031.

Schneider, T. R. \& Sheldrick, G. M. (2002). Acta Cryst. D58, 17721779.

Sheldrick, G. M. (2002). Z. Kristallogr. 217, 644-650.

Usón, I., Schmidt, B., von Bülow, R., Grimme, S., von Figura, K., Dauter, M., Rajashankar, K. R., Dauter, Z. \& Sheldrick, G. M. (2003). Acta Cryst. D59, 57-66.

Walker, R. \& Diaviacco, B. (1992). Rev. Sci. Instrum. 63, 392-395.

Weik, M., Ravelli, R., Kryger, G., McSweeney, S., Raves, M., Harel, M., Gros, P., Silman, I., Kroon, J. \& Sussman, J. (2000). Proc. Natl Acad. Sci. USA, 97, 623-628.

Weiss, M. S., Mander, G., Hedderich, R., Diederichs, K., Ermler, U. \& Warkentin, E. (2004). Acta Cryst. D60, 686-695.

Winick, H. (1995). Editor. Synchrotron Radiation Sources: A Primer. Singapore: World Scientific.

Zwart, P. H., Banumathi, S., Dauter, M. \& Dauter, Z. (2004). Acta Cryst. D60, 1958-1963. 